\title{
基于社会经济水平、人口流动和疫情防控措施 评估我国主要城市应对2019冠状病毒病的策略
}

王思思 ${ }^{1,2}$, 叶元庆 ${ }^{1,2,3}$ ，徐小林 ${ }^{1,2}$ ，王思聪 ${ }^{1,2}$ ，徐 欣 ${ }^{1,2}$ ，袁长征 ${ }^{1,2}$ ，李 舒 ${ }^{1,2}$, 曹淑殷 ${ }^{1,2}$, 李文渊 ${ }^{1,2}$, 陈 辰 ${ }^{1,2}$, 胡可嘉 ${ }^{1,2}$, 雷 浩 ${ }^{1,2}$, 朱 慧 ${ }^{4}$, 祝 勇 ${ }^{5}$, 吴息风 ${ }^{1,2,3}$

1. 浙江大学医学院附属第二医院生物统计、生物信息学和大数据中心, 浙江杭州 310009

2. 浙江大学医学院公共卫生学院大数据健康科学系, 浙江 杭州 310058

3. 浙江大学健康医疗大数据国家研究院, 浙江 杭州 310058

4. 浙江大学医学院, 浙江 杭州 310058

5. 美国耶鲁大学耶鲁公共卫生学院,康涅狄格州 纽黑文市 06520

[摘要] 目的: 评估我国主要城市社会经济水平、人口流动和疫情防控措施 对 2019 冠状病毒病(COVID-19)疫情初期发展的影响。方法: 对湖北省以外地区截 至 2020 年 2 月 19 日 COVID-19 累计确诊病例数最多的 51 个城市的 COVID-19每日 新增感染率趋势进行时间序列聚类分析, 并从社会经济水平、人口流动和疫情防 控措施三方面进行评估。结果: 在 51 个城市中共识别出 4 种不同类别的疫情发展 模式, 包括高峰模式(新余)、晚发高峰模式(甘孜州)、中高峰模式(温州等 13 个城 市) 和趋势缓和稳定模式(杭州等 36 个城市)。4 种模式以及同种模式不同城市的 指标得分分布均有差异。结论: 中国各城市的 COVID-19 疫情发展模式差异较大, 可能是受城市社会经济水平、人口流动和疫情防控措施等多方面影响。及时的卫 生应急措施和城市内部人口流动控制可能影响 COVID-19 疫情发展模式, 高风险地 区人口迁入强度对 COVID-19 累计确诊病例数有较大影响。

[关键词] 2019 冠状病毒病; 中国; 社会经济; 人口流动; 防控措施; 聚类分析 [中图分类号] R181.2 [文献标志码 ] A

\section{Impact of socioeconomic status, population mobility and control measures on COVID-10 development in major cities}

\footnotetext{
收稿日期: 2020-12-11 接受日期:2021-01-06

基金项目: 浙江大学新型冠状病毒(2019-nCoV)肺炎应急科研专项(2020XGZX003); 浙江省创新团队(2019R01007); 浙 江省重点实验室(2020E10004); 浙江省自然科学基金(LEZ20H260002)

第一作者:王思思,硕士研究生,主要从事慢性病流行病学研究; E-mail :wangsisi@zju. edu. cn; https ://orcid. org/00000003-2566-4578. 叶元庆, 研究员, 博士生导师, 主要从事遗传流行病学研究; E-mail:yuanqing99@zju. edu. cn; https:// orcid. org/0000-0001-5708-8961. 徐小林, 研究员, 博士生导师, 主要从事慢性病流行病学及防治、流行病学方法学研究; E-mail:xiaolin.xu@zju.edu. cn; https ://orcid. org/0000-0002-8203-9878

通信作者: 朱 慧, 研究员,主要从事医院管理、高教管理、社会医学等研究; E-mail:zhuhui2002@zju. edu. cn; https://orcid. org/0000-0002-8942-9916. 吴息凤,教授,博士生导师,主要从事肿瘤分子流行病学和健康医疗大数据研究; E-mail: xifengw@zju.edu.cn; https://orcid. org /0000-0003-2980-8129
} 


\section{of China}

WANG Sisi ${ }^{1,2}$, YE Yuanqing ${ }^{1,2,3}$, XU Xiaolin ${ }^{1,2}$, WANG Sicong ${ }^{1,2}$, XU Xin ${ }^{1,2}$, YUAN Changzheng $^{1,2}$, LI Shu ${ }^{1,2}$, CAO Shuyin ${ }^{1,2}$, LI Wenyuan ${ }^{1,2}$, CHEN Chen ${ }^{1,2}$, HU Kejia ${ }^{1,2}$, LEI Hao ${ }^{1,2}$, ZHU Hui ${ }^{4}$, ZHU Yong, WU Xifeng ${ }^{1,2,3}$ (1. Center for Biostatistics, Bioinformatics and Big Data, the Second Affiliated Hospital, Zhejiang University School of Medicine, Hangzhou 310009, China; 2. Department of Big Data in Health Science, School of Public Health,Zhejiang University School of Medicine, Hangzhou 310058, China; 3. National Institute for Data Science in Health and Medicine,Zhejiang University, Hangzhou 310058,China; 4. Zhejiang University School of Medicine, Hangzhou 310058, China; 5. Yale School of Public Health, Yale University, New Haven 06520, Connecticut, USA)

WANG Sisi, YE Yuangqing and XU Xiaolin contributed equally to this article.

Corresponding authors:ZHU Hui,E-mail:zhuhui2002@zju.edu.cn,https://orcid.org/ 0000-0002-8942-9916; WU Xifeng,E-mail:xifengw@zju.edu.cn,https://orcid.org/ 0000-0003-2980-8129

[Abstract] Objective:To evaluate the impact of socioeconomic status, population mobility, prevention and control measures on the early-stage coronavirus disease 2019 (COVID-19) development in major cities of China. Methods: The rate of daily new confirmed COVID-19 cases in the 51 cities with the largest number of cumulative confirmed cases as of February 19,2020 (except those in Hubei province) were collected and analyzed using the time series cluster analysis. It was then assessed according to three aspects, that is, socioeconomic status, population mobility, and control measures for the pandemic. Results: According to the analysis on the 51 cities, 4 development patterns of COVID-19 were obtained, including a high-incidence pattern (in Xinyu), a late high-incidence pattern (in Ganzi), a moderate incidence pattern (in Wenzhou and other 12 cities), and a low and stable incidence pattern (in Hangzhou and other 35 cities). Cities with different types and within the same type both had different scores on the three aspects. Conclusion: There were relatively large difference on the COVID-19 development among different cities in China, possibly affected by socioeconomic status, population mobility and prevention and control measures that were taken. Therefore, a timely public health emergency response and travel restriction measures inside the city can interfere the development of the pandemic. Population flow from high risk area can largely affect the number of cumulative confirmed cases.

[ Key words ] Coronavirus disease 2019; China; Socioeconomic status; Population flows; Control measures; Cluster analysis

[J Zhejiang Univ (Med Sci), 2021, 50(1): 52-60. ]

[缩略语] 2019 冠状病毒病 (coronavirus disease 2019,COVID-19); 国内生产总值 (gross domestic product, GDP)

目前,COVID-19大流行已成为全球性公共卫 生问题。截至 2020 年 9 月底, 全球累计确诊病例 数已经超过 3060 万, 死亡人数超过 95 万 $^{[1]}$ 。当
下 COVID-19疫苗还未普及,公共卫生措施在控 制 COVID-19 疫情蔓延中仍发挥重要作用 ${ }^{[2]}$ 。研 究发现,佩戴口罩、勤洗手、保持社交距离、咳嗽礼 
仪等个人防护措施能减缓呼吸道病毒传播 ${ }^{[3-5]}$ 。 每个城市具体的措施作为疫情控制的根本, 可有 效减少输人病例和本土病例。在 COVID-19疫情 暴发早期,国家、省、市各级有关部门采取了一系 列疫情控制措施,包括卫生应急响应、公共场所管 理、限制交通和社区管理等,这一系列措施已成功 地控制了国内的疫情 ${ }^{[6-7]}$ 。

虽然各城市采取的疫情控制措施内容类似， 但不同城市的 COVID-19 累计确诊病例数、疫情高 峰峰值和高峰时间仍存在较大差异。不同城市在 采取相似措施的情况下,形成不同疫情发展模式 的原因尚未明确。所以, 探索 COVID-19疫情控制 的关键策略将为其他疫情尚未得到缓解的国家和 地区提供循证建议, 也将为今后应对重大疫情提 供依据。本研究提出假设, 不同的疫情发展模式 可能与不同城市的社会经济水平、人口流动以及 疫情防控措施有关, 通过收集整理国内疫情初期 累计报告病例数最多的 51 个城市进行分析, 以期 验证这一假设。

\section{1 资料与方法}

\section{1 城市选择及数据来源}

2020 年 1 月 18 日湖北省武汉市报道了中国 首例 COVID-19 确诊病例, 而到 2020 年 2 月 19 日 除湖北省和香港、澳门、台湾地区以外的中国城市 每日新增病例数都已少于 5 例, 疫情基本得到控 制。因此, 本研究将疫情模式分析的时间段确定 为 2020 年 1 月 18 日至 2 月 19 日。由于湖北省 17 个城市中有 16 个城市先后采取了“封城”措施, 其 疫情发展显著区别于湖北省外的城市, 本研究选 择湖北省和香港、澳门、台湾以外地区截至 2020 年 2 月 19 日累计确诊病例数最多的 50 个城 市。由于山东省青岛市和湖南省益阳市的累计确 诊病例数同为 59 例, 并列第 50 位, 最终纳人 51 个 城市进行分析, 见表 1 。

\section{2 疫情数据来源}

51 个城市的 COVID-19 疫情数据来源于各 省/直辖市/自治区卫生健康委员会每日发布 的 COVID-19 疫情公告, 所有病例均为实验室诊断 病例。

\section{3 社会经济水平数据来源}

51 个城市的社会经济水平数据来源于各省 市统计年鉴(表 2 ), 包括常住人口数、人均 GDP, 每
千人口医生数来源于国家统计局网站 (https :// data. stats. gov. cn/easyquery. htm?cn=A01)。

\section{4 人口流动数据来源}

人口流动数据来源于百度迁徙 (http://qianxi. baidu. com), 包括武汉迁人各个城市的迁徙规 模指数和各城市的市内人口流动指数。考虑 到 COVID-19 的潜伏期为 $3 \sim 7 \mathrm{~d}^{[8-9]}$, 本研究将人口 流动数据的收集时间段确定为 2020 年 1 月 10 日 至 2 月 14 日。

基于以上两个数据, 本研究构建了 3 个人口 流动指标: (1)武汉迁人强度, 指 2020 年 1 月 10 日 至 2 月 14 日武汉迁人该城市的迁徙规模指数总 和; (2)交通管制前市内人口流动强度, 指 2020 年 1 月 10 日至 1 月 23 日的市内人口流动指数均值; (3)交通管制后市内人口流动控制指数, 指 2020 年 1 月 24 日至 2 月 14 日市内人口流动比例的均值。 市内人口流动比例为 2020 年每日市内人口流动 指数与 2019 年农历同期相比得到的比值, 市内人 口流动比例的数值越小, 代表市内人口流动控制 越好。

\section{5 疫情控制措施}

51 个城市采取的疫情控制相关措施均从各 市的人民政府网站、卫生健康委员会网站、交通运 输局网站和政府官方公众号 4 个渠道收集整理, 共包括五大类: 1)卫生应急: 成立疫情防控领导小 组/指挥部, 启动公共卫生事件一级响应; (2)公共 场所管理: 取消公众活动, 关闭公共场所 (包括电 影院、剧院、体育馆等); (3)限制武汉往来交通: 暂 停武汉/湖北交通、暂停省际客运; (4)市内交通调 整:减少市内交通、全面暂停市内交通; (5)社区封 闭管理。

\section{6 统计学方法}

应用时间序列聚类对 51 个城市的 COVID-19 疫情发展模式进行聚类分析。采用的聚类指标 为 COVID-19 每日新增感染率, 即每日新增确诊病 例数/常住人口数, 单位为 $1 / 10^{4}$ 。相似度度量采用 动态时间分配算法 ${ }^{[10]}$, 聚类算法采用 $\mathrm{K}$ 均值聚类 以进行模式识别, 数据分析由R3.6.2 中的TSclust 包实现 ${ }^{[11]}$ 。

从社会经济水平、人口流动和疫情防控措施 三个方面综合评估各城市的基础情况和特征。社 会经济水平用人口数、人均 GDP 和每千人口医生 数 3 个指标衡量; 人口流动用武汉迁人强度、交通 
表 1 截至 2020 年 2 月 19 日我国 2019 冠状病毒病累计病例数排名前 51 位城市的基本特征

Table 1 Basic characteristics of 51 cities with most cumulative cases of COVID-19 as of February 19,2020

\begin{tabular}{|c|c|c|c|c|c|}
\hline 城市 & 累计病例数 & 累计发病率 $\left(1 / 10^{4}\right)$ & 常住人口数 $\left(10^{4}\right)$ & 人均国内生产总值 (元) & 每千人医生数 \\
\hline 重庆市 & 560 & 0.1805 & 3101.79 & 65933 & 2.46 \\
\hline 浙江省温州市 & 504 & 0.5449 & 925.00 & 65055 & 3.07 \\
\hline 广东省深圳市 & 416 & 0.3193 & 1302.66 & 189568 & 2.79 \\
\hline 北京市 & 395 & 0.1834 & 2154.20 & 140211 & 5.08 \\
\hline 广东省广州市 & 339 & 0.2274 & 1490.44 & 155491 & 3.62 \\
\hline 上海市 & 333 & 0.1374 & 2423.78 & 135000 & 3.09 \\
\hline 河南省信阳市 & 269 & 0.4155 & 647.41 & 36951 & 1.86 \\
\hline 湖南省长沙市 & 242 & 0.2968 & 815.47 & 136920 & 3.78 \\
\hline 江西省南昌市 & 229 & 0.4129 & 554.55 & 95116 & 2.67 \\
\hline 黑龙江省哈尔滨市 & 194 & 0.2039 & 951.54 & 66094 & 2.79 \\
\hline 安徽省合肥市 & 173 & 0.2139 & 808.74 & 97470 & 2.76 \\
\hline 浙江省杭州市 & 169 & 0.1723 & 980.60 & 140180 & 4. 58 \\
\hline 安徽省蚌埠市 & 159 & 0.4688 & 339.20 & 50662 & 2.13 \\
\hline 河南省郑州市 & 156 & 0.1539 & 1013.60 & 101352 & 4. 15 \\
\hline 浙江省宁波市 & 156 & 0.1902 & 820.20 & 132603 & 3.17 \\
\hline 湖南省岳阳市 & 156 & 0.2691 & 579.71 & 59165 & 2.62 \\
\hline 河南省南阳市 & 155 & 0.1548 & 1001.36 & 35554 & 1.89 \\
\hline 安徽省阜阳市 & 155 & 0.1889 & 820.72 & 21589 & 1.88 \\
\hline 浙江省台州市 & 146 & 0.2378 & 613.90 & 79541 & 2.95 \\
\hline 四川省成都市 & 141 & 0.0863 & 1633.00 & 94782 & 3.77 \\
\hline 河南省驻马店市 & 139 & 0.1975 & 703.66 & 33773 & 1.88 \\
\hline 天津市 & 130 & 0.0834 & 1559.60 & 120711 & 2.75 \\
\hline 江西省新余市 & 130 & 1.0955 & 118.67 & 88500 & 2.20 \\
\hline 江西省上饶市 & 123 & 0.1806 & 681.07 & 32555 & 1.62 \\
\hline 陕西省西安市 & 120 & 0.1200 & 1000.37 & 85114 & 3.25 \\
\hline 江西省九江市 & 118 & 0.2410 & 489.68 & 55274 & 2.14 \\
\hline 安徽省毫州市 & 108 & 0.2062 & 523.72 & 24547 & 1.37 \\
\hline 江西省宜春市 & 106 & 0.1902 & 557.32 & 39199 & 1.54 \\
\hline 湖南省邵阳市 & 102 & 0.1384 & 737.05 & 24178 & 1.96 \\
\hline 广东省珠海市 & 98 & 0.5182 & 189.11 & 159428 & 3.75 \\
\hline 江苏省南京市 & 93 & 0.1102 & 843.62 & 152886 & 3.75 \\
\hline 广东省东莞市 & 92 & 0.1096 & 839.22 & 98939 & 2.33 \\
\hline 河南省商丘市 & 91 & 0.1242 & 732.53 & 32669 & 2.05 \\
\hline 江苏省苏州市 & 87 & 0.0811 & 1072. 17 & 173765 & 3.07 \\
\hline 广东省佛山市 & 84 & 0.1063 & 790.57 & 127691 & 2.53 \\
\hline 安徽省安庆市 & 83 & 0.1769 & 469.13 & 41088 & 1.89 \\
\hline 江苏省徐州市 & 79 & 0.0898 & 880.20 & 76915 & 2.93 \\
\hline 湖南省常德市 & 79 & 0.1356 & 582.72 & 58160 & 2.98 \\
\hline 湖南省株洲市 & 78 & 0.1940 & 402.08 & 65442 & 2.62 \\
\hline 河南省周口市 & 76 & 0.0876 & 867.78 & 30820 & 2.03 \\
\hline 江西省赣州市 & 76 & 0.0876 & 867.76 & 32429 & 1.73 \\
\hline 湖南省娄底市 & 76 & 0.1933 & 393.18 & 39249 & 2.41 \\
\hline 江西省抚州市 & 72 & 0.1779 & 404.72 & 34226 & 1.45 \\
\hline 福建省福州市 & 71 & 0.0917 & 774.00 & 102037 & 2.64 \\
\hline 安徽省六安市 & 69 & 0.1426 & 483.74 & 26731 & 1.91 \\
\hline 四川省甘孜州 & 67 & 0.5602 & 119.60 & 24446 & 1.41 \\
\hline 江苏省淮安市 & 66 & 0.1366 & 483.00 & 85418 & 2.72 \\
\hline 广东省中山市 & 66 & 0.1994 & 331.00 & 110585 & 2.66 \\
\hline 广东省惠州市 & 62 & 0.1233 & 502.77 & 42586 & 2.76 \\
\hline 山东省青岛市 & 59 & 0.0628 & 939.48 & 128459 & 1.71 \\
\hline 湖南省益阳市 & 59 & 0.1337 & 441.38 & 39937 & 2.53 \\
\hline
\end{tabular}

累计病例数排名不包括湖北省及香港、澳门、台湾地区. 
表 2 各省市统计数据来源网址

Table 2 Websites of provincial or city statistical yearbooks

\begin{tabular}{|c|c|}
\hline 省/城市 & 数据来源 \\
\hline 安徽省 & http://tjj. ah. gov. cn/ssah/qwfbjd/tjnj/index. html \\
\hline 北京市 & http://202.96.40.155/nj/main/2019-tjnj/zk/indexch. htm \\
\hline 重庆市 & http://tjj. cq. gov. cn//tjnj/2019/indexch. htm \\
\hline 福建省 & http://tjj. fujian. gov. cn/tongjinianjian/dz2019/contents-cn. htm \\
\hline 广东省 & http://stats. gd. gov. cn/gdtjnj/content/post_2639622. html \\
\hline 河南省 & http://www. ha. stats. gov. cn/hntj/lib/tjnj/2019/indexce. htm \\
\hline 黑龙江省 & http://www. hlj. stats. gov. cn/tjsj/tjnj/202001/t20200120_76425. html \\
\hline 湖南省 & http://222. 240. 193. 190/19tjnj/indexch. htm \\
\hline 江苏省 & http://tj. jiangsu. gov. cn/2019/indexc. htm \\
\hline 江西省 & http://www. jiangxi. gov. cn/2019jxtjnj/indexch. htm \\
\hline 山东省 & http://www. stats-sd. gov. cn/tjnj/nj2019/indexch. htm \\
\hline 陕西省 & http://tjj. shaanxi. gov. cn/upload/2020/pro/3sxtjnj/zk/indexch. htm \\
\hline 上海市 & http://tjj. sh. gov. cn/html/sjfb/202003/1004509. html \\
\hline 四川省成都市 & http://www. cdstats. chengdu. gov. cn/htm/detail_179930. html \\
\hline 四川省甘孜州 & http://tjj. gzz. gov. cn/gzztjj/tjsj/201912/742e197685484d2cb93ed39008f6a4ac. shtml \\
\hline 天津市 & http://stats. tj. gov. cn/TJTJJ434/TJGB598/TJSTJGB33/202001/t20200111_2011027. html \\
\hline 浙江省 & http://zjjemspublic. oss-cn-hangzhou-zwynet-d01-a. internet. cloud. zj. gov. cn \\
\hline
\end{tabular}

数据获取时间为 2020 年 2 月 20 日.

管制前市内人口流动强度和交通管制后市内人口 流动控制指数 3 个指标衡量; 疫情防控措施用卫 生应急、公共场所管理、限制武汉往来交通、市内 交通调整和社区管理 5 个指标衡量。将社会经济 水平和人口流动的 6 个指标重新赋值, 转换为百 分制得分。人口数、人均 GDP、每千人口医生数、 武汉迁人强度和交通管制前市内人口流动强度根 据以下公式转换: 原始值/51 个城市中该指标的最 大值 $\times 100$ 。由于交通管制后的市内人口流动控制 指数数值越小, 表示该城市人口流动控制越好, 所 以该指标采用以下公式转换: 51 个城市中该指标 的最小值/原始值 $\times 100$ 。疫情防控措施指标赋值 为每个单项措施以最早开始实施该措施的城市 计 100 分, 最晚开始的城市计 1 分, 未实施该项措 施的城市计 0 分。其中“卫生应急”分值取成立疫 情防控领导小组/指挥部和启动公共卫生事件一 级响应的平均分, “限制武汉往来交通” 分值取暂 停武汉/湖北交通和暂停省际客运中得分高 者, “市内交通调整”分值取减少市内交通和全面 暂停市内交通中得分高者。最后, 将 5 个措施指 标得分相加并转化为百分制得到疫情防控措施指 标综合得分。

\section{2 结 果}

2.151 个城市疫情发展模式聚类分析结果 聚类分析结果显示, 51 个城市的疫情发展模
式可分为 4 类: 高峰模式 (类 1 )、晚发高峰模式 (类 2)、中高峰模式 (类 3 ) 和趋势缓和稳定模式 (类 4), 结果如图 1所示。类 1 (红色线条) 和类 2 (绿色线条)各有一个城市, 分别为新余市和甘孜 州。类 1 (新余市) 的疫情峰值远高于其他 3 类, 新 增感染率峰值为其余地区的 2 倍以上, 前期迅速 上升,后期缓慢下降至其他城市同等水平。类 2 (甘孜州) 前期仅有少数病例, 到 2 月中下旬其他 地区都呈下降趋势时反而开始上升。36个疫情发 展缓和平稳的城市被归为类 4 (黄色线条), 包括北 京、上海、广州、杭州、成都、重庆、株洲、安庆等。 其余 13 个城市为类 3 (蓝色线条), 包括长沙、南 昌、宁波、深圳等。

2.251 个城市社会经济情况、人口流动和疫情 防控措施综合评价

图 2展示了不同疫情发展模式城市在社会经 济水平、人口流动和疫情防控措施三方面 12 项指 标的得分分布情况。 4 类城市的指标得分分布存 在明显差异,同一类中不同城市得分分布差别也 较大。51 个城市各项疫情控制措施的实施时间如 附表 1所示, 社会经济水平、人口流动各项指标及 疫情防控措施得分见附表 2 。

在所有城市中, “卫生应急”得分最高的是 类 4 的杭州。杭州在 2020 年 1 月 18 日还未出现 确诊病例的情况下迅速行动, 开展了疫情防控相 关的部署和工作,而其他城市普遍在 2020 年 1 月 


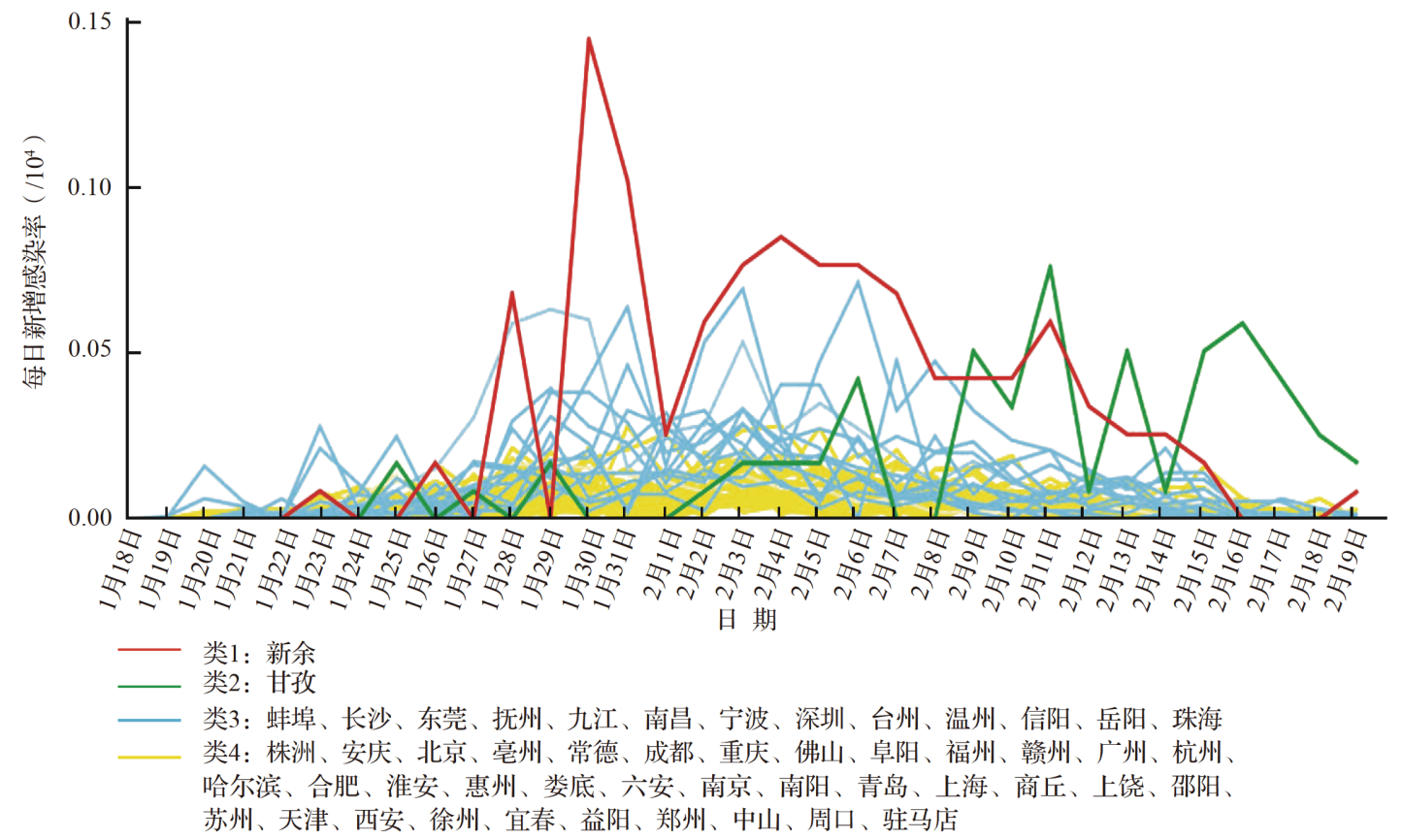

图 12020 年 1 月 18 日至 2 月 19 日我国 51 个城市 2019 冠状病毒病疫情发展模式聚类分析结果

Figure 1 Results of cluster analysis of daily COVID-19 incidence rate in 51 cities from January 18 to February 19,2020

20 日之后成立领导小组, 1 月 23 日之后启动一级 响应。51 个城市“公共场所管理”措施的开展时间 为 2020 年 1 月 23 日至 1 月 28 日, 时间较为接近。 其中类 3 的长沙、深圳、珠海,类 4 的北京、东莞、广 州、南阳在 2020 年 1 月 23 日开始实施, 得分最 高。“限制武汉往来交通”措施包含暂停武汉/湖 北交通和暂停省际客运两种类型, 有些城市如阜 阳采取的是先暂停武汉/湖北的交通, 后续强化控 制力度全面暂停省际客运的方式; 也有一些城市 如周口直接暂停了所有省际客运。在 51 个城市 中, “限制武汉往来交通”最及时的是类 4 的阜阳, 在 2020 年 1 月 21 日武汉还未封城之前就暂停了 与武汉/湖北的客运, 有效减少了前期的病例输 人, 武汉输人强度得分仅为 23.93 分, 低于大多数 地区。将五项措施合并后疫情防控措施总分最高 的是类 4 的北京。北京在 “公共场所管理”为 100 分, 并且其他四项措施得分均处于中上水平。

\section{3 讨 论}

本研究采用聚类分析和综合评价的方法, 从 城市的社会经济水平、人口流动和疫情控制措施 三个方面综合评估有助于遏制 COVID-19 传播的 因素, 研究结果表明及时、全方位的疫情控制策略 对疫情控制具有重要意义。
目前已有部分研究评估了各种疫情控制措施 的有效性 ${ }^{[12-15]}$ 。牛津大学针对国家层面措施情况 开发了牛津 COVID-19 政府反应追踪器以记录和 比较不同国家的防控措施 ${ }^{[16]}$ 。此外, Qiu 等 ${ }^{[17]}$ 和 Sajadi 等 ${ }^{[18]}$ 研究了社会经济学因素和天气特征 对 COVID-19传播的影响。区别于其他研究着眼 于单一因素, 本研究旨在综合评估多方面因素 对 COVID-19 疫情发展模式的影响, 并探索对疫情 控制有效的因素。研究结果表明,多方面因素对 疫情发展模式的影响是综合而复杂的。如图 2 所 示, 部分城市具有相似的 COVID-19 疫情发展模式 (类 3 和类 4 ), 但其社会经济水平、人口流动和疫 情控制措施 12 个指标得分分布明显不同。

本文资料显示,全面综合的疫情防控策略对 遏制 COVID-19 疫情蔓延十分必要。一项针对武 汉市疫情的研究发现, 2020年 1 月 26 日之前武汉 市 COVID-19 的有效再生数高达 3.0 , 疫情处于快 速上升阶段。而在实施了多方面的公共卫生措施 后,到 2020 年 2 月 6 日武汉市 COVID-19 的有效再 生数已降至 1.0 以下,证实了公共卫生措施的有 效性 ${ }^{[19]}$ 。本文资料显示, 大多数城市在 2020 年 2 月中旬前都采取了包括“卫生应急”、“公共场 所管理”、“限制武汉往来交通”、“市内交通调整”和 “社区封闭管理”在内的多方面的疫情控制措施。 
类1
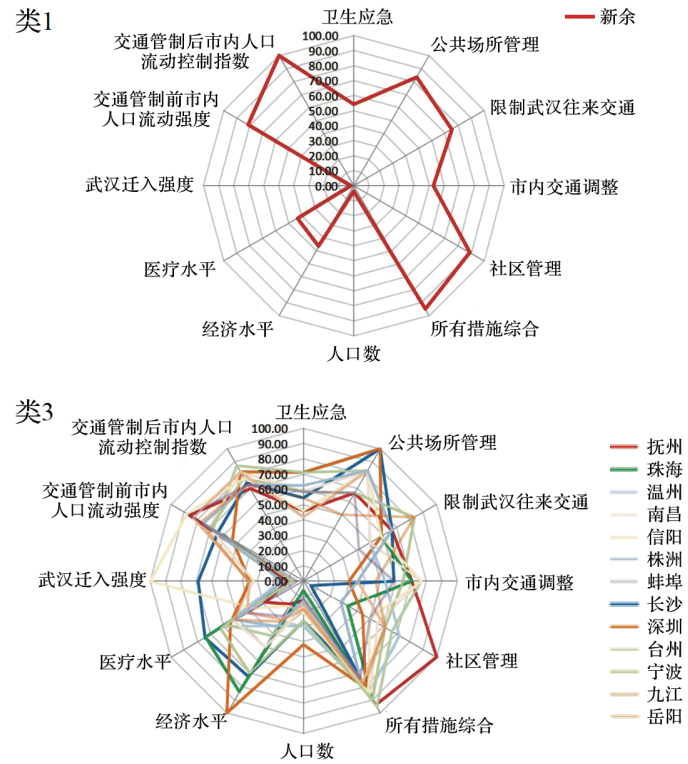

类2
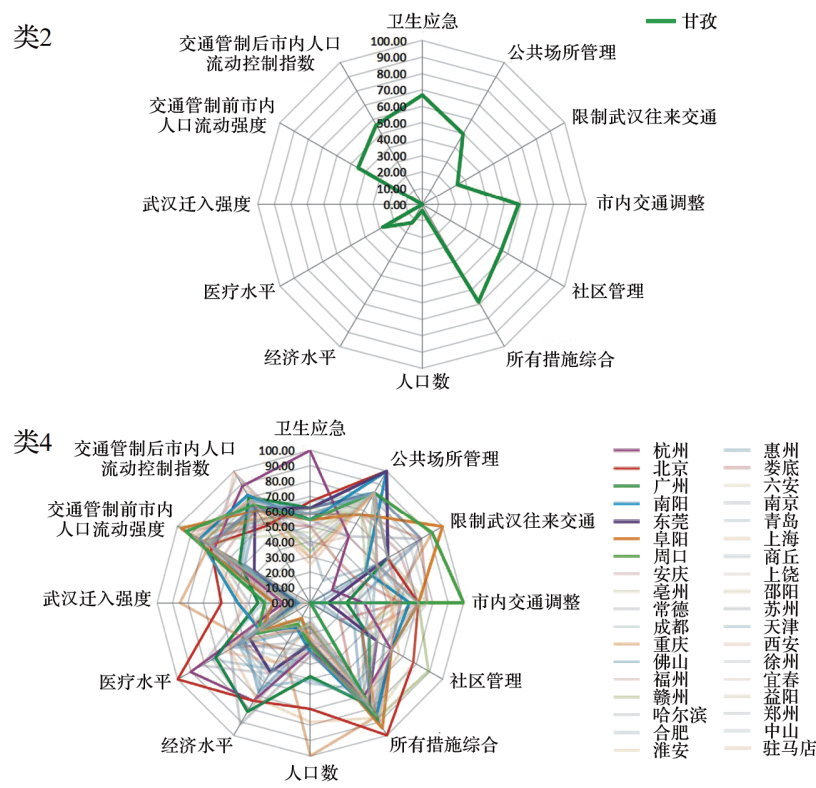

图 2 我国 4 类疫情发展模式城市 12 个指标得分雷达图

Figure 2 Radar maps showing the scores of 12 indicators of cities among 4 city clusters

虽然各城市的社会经济水平、人口流动和措施实 施日期不一,但在采用综合疫情控制策略后,各城 市每日新增病例数在 2020 年 2 月 19 日均已少 于 5 例,疫情蔓延形势基本得到控制。

本研究结果显示, 及时的卫生应急可能是影 响 COVID-19疫情发展的重要因素。启动公共卫 生事件一级响应标志着地方政府开始根据国务院 的统一部署和指挥, 全力组织协调公共卫生应急 工作。当地卫生行政部门、疾控中心、医疗卫生机 构和其他相关部门进行联防联控, 全面协同开展 公共卫生疫情防控,开展包括病例搜索、确诊病例 隔离治疗、密切接触者和高风险地区来源个体隔 离观察、公共场所体温检测、加强卫生宣教等一系 列措施。在本研究纳人的 51 个城市中, 有 5 个城 市 (杭州、东莞、甘孜、徐州和商丘) 在本市确诊首 例 COVID-19 之前就启动了公共卫生事件一级响 应。杭州是在发现第 1 例 COVID-19病例 $3 \mathrm{~d}$ 前启 动响应的,其他 4 个城市均提前 $1 \mathrm{~d}$ 。对应这几个 城市的疫情发展模式,杭州、东莞、徐州、商丘均属 于疫情缓和平稳的类 4 , 而甘孜州在 2 月份前也仅 有少数确诊病例。已有研究表明, 与未提前采取 措施的城市相比, 提前采取预防措施的城市在疫 情暴发第一周的确诊病例数减少 $33.3 \%(95 \% C I$ : $11.1 \% \sim 44.4 \%)^{[13]}$ 。因此, 组织严密的控制措施、 充足的人力和物资准备在 COVID-19疫情控制初 期发挥了关键作用。
另外, 城市内部人口流动控制可能是遏 制 COVID-19疫情发展的关键因素。严重急性呼 吸综合征冠状病毒 2 主要通过呼吸道飞沫和接触 传播, 上下班等短距离人口流动与流感等呼吸道 传染病的区域传播相关 ${ }^{[20]}$ 。研究显示, 武汉封城 和城市间的交通限制有效减少了其他城市的输人 病例 ${ }^{[12-13,21]}$, 而城市内部分人口流动限制对控制 疫情传播也很重要。本研究中, 甘孜州尽管在首 例确诊病例报告前就启动了一级响应, 前期疫情 缓和, 但在 2020 年 2 月份其他城市新增病例数逐 渐减少的情况下, 甘孜州的疫情出现晚发高峰。 与其他 4 个提前采取措施城市的主要差异在于城 市内部人口流动控制, 甘孜州在 2020 年 1 月底 和 2 月初的市内人口流动比例与其他 50 个城市相 比处于较高水平, 因此市内人口流动控制不佳可 能是甘孜州疫情晚发高峰的原因。市内人口流动 控制情况是公共场所管理、市内交通调整、社区封 闭管理和其他社交距离控制措施的综合结果,这 些措施可有效减少城市内人员流动,继而遏制 COVID-19 在城市内的进一步扩散和恶化。

本研究结果还提示, 武汉迁人强度对城 市 COVID-19 累计病例数有很大影响。例如岳阳 和常德这两个城市具有相似的人口数、经济水平、 医疗水平、交通管制前市内人口流动强度和措施 总得分, 但岳阳在武汉迁人强度的得分高于常德 (分别为 35.81 和 22.64 ), 最终岳阳 2020 年 2 月 19 
日累计病例数几乎是常德的2倍(岳阳156例, 常德 79 例)。这一发现与其他研究的结果一致 ${ }^{[21-22]}$ 。因 此, 针对当前 COVID-19 的全球疫情, 建议各地区 应限制高风险地区的人口流人。

本研究的优势在于采用了多个指标综合评估 可能会影响 COVID-19 疫情发展趋势的因素。此 外, 在研究城市间人口流动的同时还纳人了城市 内部的人口流动, 综合考虑了一代输人病例和二 代病例的情况。但本研究存在几个不足。首先, 为保证 51 个城市之间的一致性, 我们统一从 4 个 官方渠道收集措施信息, 可能未包含所有措施。 其次, 本研究假设每个城市措施的实行力度相似, 聚焦于措施实施的及时性对各城市疫情控制措施 进行赋值。为了弥补这一不足, 我们通过比 较 2020 年和 2019 年农历同期的城市内人口流动 强度来分析城市内人口流动控制强度, 这一指标 可以在一定程度上反映控制措施的力度和效果。

综上, 中国各城市 COVID-19 疫情发展模式 存在差异, 可能是受到社会经济水平、人口流动和 疫情防控措施等多方面的影响, 及时的卫生应急 和城市内部人口流动控制可能影响 COVID-19 的 发展模式, 高风险地区人口迁人强度对 COVID-19 累计确诊病例数有较大影响, 因此限制高危地区 的人口迁人是十分必要的。

\section{参考文献}

[1] World Health Organization. Coronavirus disease (COVID$2019)$ situation report [EB/OL]. (2020-08-30) [202009-20]. https://www. who. int/docs/default-source/ coronaviruse/situation-reports/20200831-weekly-epiupdate-3. pdf?sfvrsn=d7032a2a_4.

[2] Report of the WHO-China joint mission on coronavirus disease 2019 (COVID-19) [EB/OL]. (2020-02-28) [2020-03-05]. https://www. who. int/publications-detail/report-of-the-who-china-joint-mission-on-coronavirus-disease-2019-( covid-19).

[3] SAUNDERS-HASTINGS P, CRISPO J A G, SIKORA L, et al. Effectiveness of personal protective measures in reducing pandemic influenza transmission: A systematic review and meta-analysis[J]. Epidemics, 2017,20:120. DOI : 10. 1016/j. epidem. 2017. 04. 003.

[4] AHMED F, ZVIEDRITE N, UZICANIN A. Effectiveness of workplace social distancing measures in reducing influenza transmission:a systematic review [J]. BMC Public Health, 2018,18(1):518. DOI: 10. 1186/s12889-018-5446-1.

[5] AiEllo A E, COULBORN R M, ARAGON T J, et al.
Research findings from nonpharmaceutical intervention studies for pandemic influenza and current gaps in the research[J]. Am J Infection Control, 2010,38(4) : 251-258. DOI: 10. 1016/j. ajic. 2009. 12. 007.

[6] WU Z, MCGOOGAN J M. Characteristics of and important lessons from the coronavirus disease 2019 (COVID-19) outbreak in China[J]. JAMA, 2020,323 (13) : 1239. DOI: 10. 1001/jama. 2020. 2648.

[7] DENG S Q, PENG H J. Characteristics of and public health responses to the coronavirus disease 2019 outbreak in China $[\mathrm{J}]$. JCM, 2020,9(2):575. DOI: $10.3390 /$ jcm9020575.

[8] LIANG W H, GUAN W J, LI C C, et al. Clinical characteristics and outcomes of hospitalised patients with COVID-19 treated in Hubei (epicentre) and outside Hubei (non-epicentre): a nationwide analysis of China $[J]$. Eur Respir J, 2020,55(6) : 2000562. DOI: $10.1183 / 13993003.00562-2020$.

[9] YANG Y,LU Q,LIU M,et al. Epidemiological and clinical features of the 2019 novel coronavirus outbreak in China $[\mathrm{J} / \mathrm{OL}]$. medRxiv, 2020. DOI:10.1101/ 2020. 02. 10. 20021675.

[10 ] RAKTHANMANON T, CAMPANA B, MUEEN A, et al. Addressing big data time series : mining trillions of time series subsequences under dynamic time warping [C]. ACM Trans Knowl Discov Data, 2013,7(3): 1-31. DOI: 10. 1145/2500489.

[11] MONTERO P, VILAR J A. TSclust : an $R$ package for time series clustering $[J]$. J Stat Soft, 2014,62 (1) :1-43. DOI: $10.18637 /$ jss. v062. i01.

[12] CHINAZZI M, DAVIS J T, AJELLI M, et al. The effect of travel restrictions on the spread of the 2019 novel coronavirus (COVID-19) outbreak [J/OL]. Science, 2020,368(6489):395-400. DOI:10. 1126/science. aba9757.

[13] TIAN H, LIU Y, LI Y, et al. An investigation of transmission control measures during the first 50 days of the COVID-19 epidemic in China $[\mathrm{J}]$. Science, 2020,368(6491):638-642. DOI: 10. 1126/science. abb6105.

[14] KRETZSCHMAR M, ROZHNOVA G, VAN BOVEN

M. Isolation and contact tracing can tip the scale to containment of COVID-19 in populations with social distancing $[\mathrm{J} / \mathrm{OL}]$. SSRN J, 2020. DOI:10.2139/ ssrn. 3562458 .

[15] ZHOU X, WU Z, YU R, et al. Modelling-based evaluation of the effect of quarantine control by the Chinese government in the coronavirus disease 2019 outbreak [J]. Sci China Life Sci, 2020,63(8) : $1257-$ 1260. DOI: 10. 1007/s11427-020-1717-9.

[16] Oxford COVID-19 government response tracker. [2020-05-10]. https://www. bsg. ox. ac. uk/research/research-projects/oxford-covid-19-govern- 
ment-response-tracker.

[17] QIU Y, CHEN X, SHI W. Impacts of social and economic factors on the transmission of coronavirus disease 2019 (COVID-19) in China[J]. J Popul Econ, 2020,33(4):1127-1172. DOI : 10. 1007/s001 48-020-00778-2.

[18] SAJADI M M, HABIBZADEH P, VINTZILEOS A, et al. Temperature, humidity and latitude analysis to predict potential spread and seasonality for COVID-19 [J]. SSRN J, 2020. DOI: 10. 2139/ssrn. 3550308.

[19] PAN A, LIU L, WANG C, et al. Association of public health interventions with the epidemiology of the COVID-19 outbreak in Wuhan,China[J]. JAMA, 2020,323(19): 1915. DOI: 10. 1001/jama. 2020. 6130.

[20] VIBOUD C, BJØRNSTAD O N, SMITH D L, et al.
Synchrony, waves, and spatial hierarchies in the spread of influenza[J]. Science, 2006,312(5772): 447-451. DOI: 10. 1126/science. 1125237.

[21] LIU K, AI S, SONG S, et al. Population movement, city closure in Wuhan and geographical expansion of the 2019-nCoV pneumonia infection in China in January 2020[J]. Clin Infect Dis, 2020,71(16): 20452051. DOI: $10.1093 / \mathrm{cid} / \mathrm{ciaa} 422$.

[22] CAO Z,ZHANG Q, LU X, et al. Incorporating human movement data to improve epidemiological estimates for 2019-nCoV [J/OL]. medRxiv, 2020. DOI: 10. 1101/2020. 02. 07. 20021071.

[本文编辑 沈 敏 余 方] 